\title{
Long-Lived Mesoscopic Entanglement outside the Lamb-Dicke Regime
}

\author{
M. J. McDonnell, J. P. Home, D. M. Lucas, G. Imreh, B. C. Keitch, D. J. Szwer, N. R. Thomas, \\ S. C. Webster, D. N. Stacey, and A. M. Steane \\ Clarendon Laboratory, Department of Physics, University of Oxford, Parks Road, Oxford OX1 3PU, United Kingdom
} (Received 15 May 2006; published 7 February 2007)

\begin{abstract}
We create entangled states of the spin and motion of a single ${ }^{40} \mathrm{Ca}^{+}$ion in a linear ion trap. We theoretically study and experimentally observe the behavior outside the Lamb-Dicke regime, where the trajectory in phase space is modified and the motional coherent states become squeezed. We directly observe the modification of the return time of the trajectory, and infer the squeezing. The mesoscopic entanglement is observed up to $\Delta \alpha=5.1$ with coherence time $170 \mu$ s and mean phonon excitation $\bar{n}=16$.
\end{abstract}

DOI: 10.1103/PhysRevLett.98.063603

A two-state system interacting with a quantum harmonic oscillator has for a long time played a fundamental role in quantum optics, and more recently has attracted interest in the context of micromechanical oscillators [1], quantum information [2], and mesoscopic quantum physics. The states of motion of a quantum oscillator which most closely resemble classical states of motion are the Glauber coherent states. It has been pointed out that a superposition of such states, with a large difference between their coherent state parameters $\alpha$, permits an investigation of mesoscopic quantum physics using this system [3,4]. In particular, interest has focused on a superposition involving an entanglement between the large system (the oscillator) and a smaller (e.g., two-state) system, viz:

$$
|\psi\rangle=\frac{1}{\sqrt{2}}\left(\left|\alpha_{\uparrow}\right\rangle|\uparrow\rangle+e^{i \varphi}\left|\alpha_{\downarrow}\right\rangle|\downarrow\rangle\right),
$$

where $\Delta \alpha^{2}=\left|\alpha_{\uparrow}-\alpha_{\downarrow}\right|^{2}$ is large, $|\uparrow\rangle,|\downarrow\rangle$ are the states of the two-state system, and the interference phase $\varphi$ must be stable and under control in the experiment (as must the values of $\alpha_{\uparrow, \downarrow}$, including their relative phase).

The coherent states $\left|\alpha_{\downarrow, \dagger}\right\rangle$ are mesoscopic in that their energy is $\bar{n}=|\alpha|^{2} \gg 1$ in units of the fundamental excitation energy (the gap between ground and excited states) and the separation $x_{s}$ of the motional wave packets is greater than their individual size $x_{0}$ by the ratio $x_{s} / x_{0}=$ $2 \Delta \alpha \gg 1$. The size of the Hilbert space required to express the motional state is approximately $\log _{2} \bar{n}$ qubits, and in the case of a state such as (1) there is entanglement with another degree of freedom. The phase coherence time, $T_{2}$, is sensitive to the separation [5]. These measures are summarized by the list $\left\{\bar{n}, \Delta \alpha, x_{s}, T_{2}\right\}$.

States of the type (1) have been realized in a number of systems. For experiments where the coherence of the two parts of the state was observed [6], the reported parameter values were $\left\{\bar{n}, \Delta \alpha, x_{s}, T_{2}\right\}=\{8.8,2.97,42 \mathrm{~nm}, O(10 \mu \mathrm{s})\}$ [3], \{12, 5.2, $73 \mathrm{~nm}, 6 \mu \mathrm{s}\}$ [9]; $\{1,2,14 \mathrm{~nm}, \sim 0.5 \mathrm{~ms}\}$ [7]; $\{9.5,1.8,-, 90 \mu \mathrm{s}\}[4]$.

We present experiments in which the mesoscopic superposition state (MSS) is realized with large values of both
PACS numbers: 42.50.Dv, 03.65.Ta, 03.65.Ud, 03.67.Mn

the size and the coherence time together, and we describe and demonstrate a qualitatively new behavior which appears outside the Lamb-Dicke regime (LDR). The LDR is the regime where the extent of the particle motion is small compared to the distance over which the applied forces vary. We have generated MSS's of the spin and motion of a trapped ${ }^{40} \mathrm{Ca}^{+}$ion with $\left\{\bar{n}, \Delta \alpha, x_{s}, T_{2}\right\}=$ $\{16,5.1,170 \mathrm{~nm}, \simeq 170 \mu \mathrm{s}\}$. The Hilbert space dimension is approximately 5 qubits. In our experiments the driving of the motion goes outside the LDR, resulting in a dramatic modification of the trajectory in phase space and squeezing of the coherent state [10]. We achieve MSS's of coherent states, and also infer superpositions of squeezed states with a squeezing parameter (ratio of principal axes of the Wigner function $) \simeq 3$.

Our system consists of a single spin-half particle in a harmonic potential, subject to a "walking wave" of light formed by counterpropagating laser beams in a standing wave configuration with a frequency difference applied between the two beams. The walking wave provides a spin-dependent force on the particle. The interaction Hamiltonian is $H_{I}=H_{\pi}+\sum_{m} V_{m}|m\rangle\langle m|$, where $m=\uparrow, \downarrow$

$$
V_{m}=\hbar \Omega \cos \left(k \hat{x}-\omega t+\phi_{m}\right),
$$

and $H_{\pi}=\hbar \Delta_{\pi}(|\uparrow\rangle\langle\uparrow|-| \downarrow\rangle\langle\downarrow|) / 2 . V_{m}$ is a light shift from far-off-resonant single-photon excitation; $H_{\pi}$ is a light shift from off-resonant Raman excitation of spin-flip transitions. The latter has no effect on the motion, but causes the spin state to precess. $k$ and $\omega$ are the wave vector and frequency of the walking wave.

The position dependence of $V_{m}$ results in a spindependent force $f_{m}(x, t)=-d V_{m} / d x$. The classical equation of motion is $2 M \omega_{0} x_{0}(d \alpha / d t)=i \exp \left(i \omega_{0} t\right) f_{m}(x, t)$ where $\alpha=\exp \left(i \omega_{0} t\right)\left(x+i p / M \omega_{0}\right) / 2 x_{0}$, and $x_{0}$ is a length scale. $M$ is the mass of the ion and $\omega_{0}$ its natural oscillation frequency in the trap. If we take $x_{0}=$ $\left(\hbar / 2 M \omega_{0}\right)^{1 / 2}$ then $\alpha$ corresponds exactly to the coherent state parameter in the quantum treatment.

We consider motional states $|\alpha\rangle$ starting at or near $\alpha=$ 0 . For small $|\langle k x\rangle|$ we have the LDR, where the force 
$f_{m}(x, t) \simeq \hbar \Omega k \sin \left(\omega t-\phi_{m}\right)$ is independent of position. In this case an analytical solution of the time-dependent Schrödinger equation (TDSE) is possible [11,12]. The quantum state is merely displaced along a trajectory $\alpha(t)$ exactly matching the classical prediction. For $|\delta| \ll \omega_{0}$, where $\delta=\omega-\omega_{0}, \alpha(t)$ describes a circle of diameter $\eta \Omega / \delta$, where $\eta=k x_{0}$ is the Lamb-Dicke parameter. The motion around the circle is at a constant rate, completing a loop at integer multiples of $2 \pi / \delta$. The quantum state picks up a phase proportional to the area of the loop, plus a contribution $\pm \Delta_{\pi} t / 2$, and an oscillating phase from $\int V_{m} d t$. This oscillating phase scales as $1 / \eta$; it is important when $\langle k x\rangle \ll 1$ but is small outside the LDR when $\langle k x\rangle \sim 1$ and we will ignore it hereafter.

We studied the dynamics outside the LDR using numerical integration of the classical equation of motion, and an approximate numerical solution of the TDSE. The latter included up to $n_{\max }=100$ harmonic oscillator levels and terms in all orders of $\eta$ for the carrier and first three motional sidebands in $H_{I}$.

The position dependence of the force causes the trajectory to be noncircular and the motional state is squeezed. For modest values of $\langle k x\rangle$ we find that the squeezing is negligible and the quantum wave packet simply follows the modified classical trajectory. For larger values the wave packet is squeezed, changing shape dramatically for longer times with multiple loops in phase space. Figure 1 shows an illustrative example. The departure from a circular trajectory and the squeezing are clearly seen. Note that $\alpha(\tau)$, where $\tau$ is the length of time that the force is applied, still returns to the origin, but at a time $t_{r}$ earlier than the value $2 \pi / \delta$ for a state which stays within the LDR.

We find $\alpha(\tau)$ begins to differ clearly from a circle when $\left\langle k x_{\max }\right\rangle=2 \eta\left|\alpha_{\max }\right|>1$. Each loop is shaped like a tear drop and $t_{r}$ is reduced. In the early stages of the motion, it is still within the LDR so the initial behavior both in terms of amplitude and phase is accurately described by the simple analytical treatment outlined above. Similarly, when the amplitude of the motion drops for the first time back into the LDR the analytical treatment again gives a good representation of the behavior, if one takes into account the difference between the actual and LDR return times.

We experimentally investigated the behavior using a single ${ }^{40} \mathrm{Ca}^{+}$ion in a linear ion trap [13]. The two-state system is the spin- $1 / 2$ ground state of the ion, and the potential (2) is realized by the light shift when the ion is illuminated by a laser walking wave produced by a $60^{\circ}$ pair of laser beams, both far $(30 \mathrm{GHz})$ detuned from the $\lambda=$ $397 \mathrm{~nm} S_{1 / 2}-P_{1 / 2}$ transition, and with difference wave vector $k=2 \pi / \lambda$ along the trap axis $x$. Their difference frequency $\omega$ is generated with $1 \mathrm{~Hz}$ precision by acoustooptic modulation. A quantization axis is defined by a 1.4 Gauss magnetic field $\mathbf{B}$ at $57^{\circ}$. Both the quantization and trap axes are horizontal. The polarization of each beam is

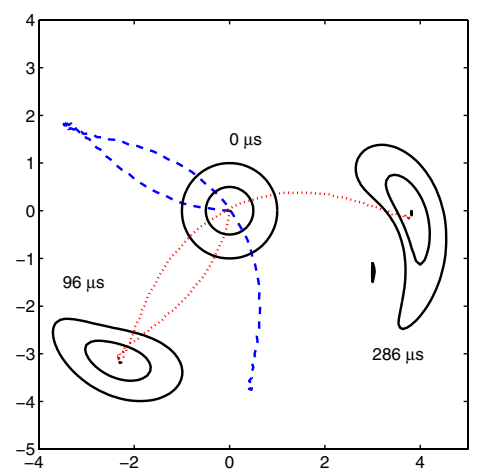

FIG. 1 (color online). Trajectories in phase space $\alpha(\tau)$ (dotted and dashed lines) and motional Wigner function (contour lines at 0,1,2 standard deviations) for the parameter values $\omega_{0} / 2 \pi=$ $536 \mathrm{kHz}, \eta=0.244, \Omega / 2 \pi=93 \mathrm{kHz}, \delta / 2 \pi=3.4 \mathrm{kHz}$ and varying forcing time $\tau$. The trajectories are shown for the two spin states for $\Phi_{w}=1.41$, up to the time $2 \pi / \delta=286 \mu \mathrm{s}$. $\Phi_{w}=\left|\phi_{\uparrow}-\phi_{\downarrow}\right|$ is the phase angle between the forces on the two spin states. The return time is $t_{r}=192 \mu \mathrm{s}$. For clarity, the Wigner function is shown for just one of the trajectories at three example times, $\tau=0,96$, and $286 \mu \mathrm{s}$. The squeezing is $\simeq 3$ at $t_{r} / 2$. In the experiments, superpositions of motion along both members of such pairs of trajectories are created.

set close to linear at the ion, by nulling any differential light shift observed in Ramsey experiments. One beam is horizontally polarized, the other at $69^{\circ}$ to the vertical. The resulting light field has three components: a $\sigma^{+}$polarized walking wave, a $\sigma^{-}$polarized walking wave, and a predominantly $\pi$ polarized traveling wave. The transition in the ion is $J=\frac{1}{2} \rightarrow \frac{1}{2}$, so the $\sigma^{+}\left(\sigma^{-}\right)$light interacts with $|\downarrow\rangle(|\uparrow\rangle)$, giving rise to $V_{\downarrow},\left(V_{\uparrow}\right)$, respectively.

The ion is first prepared in $|\downarrow\rangle \rho\left(\bar{n}_{0}\right)$, where $\rho\left(\bar{n}_{0}\right)$ is a thermal motional state close to the ground state with mean motional state occupation number $\bar{n}_{0}$ [14]. A sequence of laser pulses is then applied, and finally the spin state is measured by selective shelving followed by fluorescence detection, see [15]. This is repeated 500 times to accumulate statistics, then a parameter value is changed and the sequence repeated.

Initial experiments were carried out by Ramsey interferometry, with the oscillating force pulse $\mathcal{W}$ applied in the gap. The $\pi / 2$ pulses were Raman transitions driven by the walking wave, with tunable relative azimuthal phase $\phi$. The data reported here were obtained using a spin-echo sequence, to eliminate slow phase noise. The first $\pi / 2$ pulse evolves the spin to $(|\uparrow\rangle+|\downarrow\rangle) / \sqrt{2}$. After the $\mathcal{W}$ pulse, of duration $\tau$, the system is (up to a global phase) in the state (1), with $\varphi=\Delta_{\pi} \tau$. In the LDR, $\left|\alpha_{\uparrow, \downarrow}\right\rangle$ are coherent states, and outside this limit they are squeezed or more general states, centered in phase space close to $\alpha_{\uparrow, \downarrow}$. The observed signal after the final pulse is

$$
P(\uparrow)=\left(1-\operatorname{Re}\left[\left\langle\alpha_{\uparrow} \mid \alpha_{\downarrow}\right\rangle e^{i\left(\phi-\Delta_{\pi} \tau\right)}\right]\right) / 2 .
$$

We determine $\left\langle\alpha_{\uparrow} \mid \alpha_{\downarrow}\right\rangle$ by observing $P(\uparrow)$ as a function of $\phi$ 
TABLE I. Experimental parameters and results. Column 1 gives the data set number. The next 5 columns give values extracted directly from the fringe data by curve fitting. The rest of the Table gives further raw information and derived quantities. $\eta$ is known from the trap frequency. $\Omega_{c}$ is obtained by Rabi flopping on the carrier (value a) and from the fitted $\Delta_{\pi}$ (value b). The detuning $\delta$ is set experimentally to within $0.5 \mathrm{kHz}$ for a given data set (c) and can be evaluated also (via the TDSE) from the data analysis (d). The same process leads to a value of $\alpha_{0}$ (f) which can be compared with that deduced from the parameters of the light field (e). Finally, we infer $\alpha_{\max }$, the maximum excursion in phase space, and $\Delta \alpha_{\max }$, the maximum distance in phase space between the two spin components, from the TDSE. We estimate b,d, f, $\alpha_{\max }$, and $\Delta \alpha_{\max }$ have 5\% accuracy; the consistency check a,c, and e combines Rabi flopping, relative power, and polarization measurements and is accurate to $\sim 15 \%$.

\begin{tabular}{rrrrrrrrrrrrrrrrr}
\hline \hline Set & $D$ & $t_{r}(\mu \mathrm{s})$ & $\gamma\left(\mathrm{ms}^{-1}\right)$ & $B$ & $\Delta_{\pi} / 2 \pi(\mathrm{kHz})$ & $\bar{n}_{0}$ & $\eta$ & $\Omega_{c} / 2 \pi(\mathrm{kHz})$ & $\delta / 2 \pi(\mathrm{kHz})$ & $\alpha_{0}$ & $\alpha_{\max }$ & $\Delta \alpha_{\max }$ \\
\hline & & & & & & & & & $\mathrm{a}$ & $\mathrm{b}$ & $\mathrm{c}$ & $\mathrm{d}$ & $\mathrm{e}$ & $\mathrm{f}$ & & \\
1 & 1.45 & 89 & 2.0 & 2.15 & 4.48 & 0.07 & 0.244 & 139 & 145 & 10 & 10.1 & 2.2 & 2.4 & 2.1 & 2.7 \\
2 & 2.27 & 147 & 4.1 & 3.24 & 4.49 & 0.07 & 0.244 & 139 & 145 & 5 & 5.3 & 4.5 & 4.2 & 3.1 & 4.0 \\
3 & 3.12 & 192 & 5.6 & 4.27 & 4.46 & 0.07 & 0.244 & 139 & 145 & 3.5 & 3.4 & 6.4 & 6.8 & 4.0 & 5.1 \\
4 & 1.50 & 91 & 3.5 & 2.03 & 7.36 & 0.04 & 0.199 & 151 & 185 & 10 & 10.2 & 2.0 & 2.3 & 2.1 & 2.7 \\
5 & 1.88 & 160 & 4.6 & 2.72 & 4.27 & 0.02 & 0.245 & 137 & 142 & -5.5 & -5.2 & 4.0 & 3.4 & 2.7 & 3.5 \\
\hline \hline
\end{tabular}

and $\tau$. For each value of $\tau$ we accumulate the interference fringe pattern as a function of $\phi$, and fit it with a sinusoid. To factor out the effect of magnetic field noise, we normalize the observed fringe amplitude by comparison to that obtained in a control experiment, having exactly the same timing but with no $\mathcal{W}$ pulse. The amplitude of the control experiment fringes drop to typically $40 \%$ for a $300 \mu$ s spin-echo time. Experimental parameters and results are shown in Table I.

The observed amplitude $A$ and phase offset $\phi_{0}$ of the fringes are shown as functions of $\tau$ for various data sets in Figs. 2 and 3. This information enables us to infer the evolution of the system. The analysis is simplified by the fact that critical data exist only where the motion is within the LDR; outside this region the fringe amplitude is close to zero. To fit $A$ we can therefore use a LDR expression [5], modified to take account of the reduction in return time:

$$
A(\tau)=e^{-\gamma \tau} \exp \left[-2 D^{2} \sin ^{2}\left(\pi \tau / t_{r}\right)\right]
$$

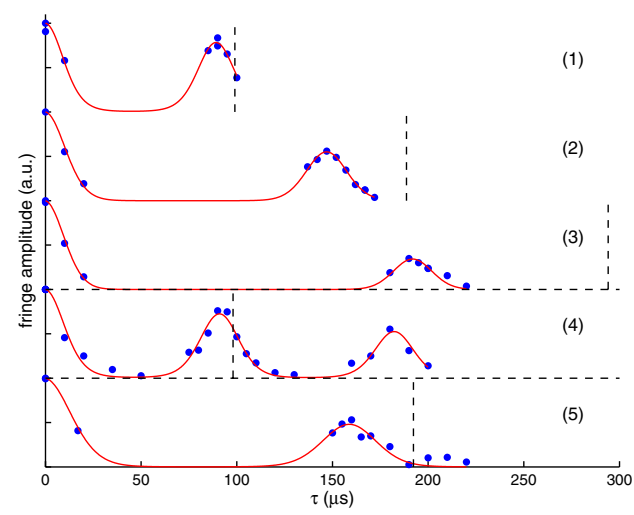

FIG. 2 (color online). Observed fringe amplitudes as a function of $\tau$, normalized to the value at $\tau=0$. Each point is obtained from a sinusoidal fit to a scan over $\phi$. Dashed horizontal lines separate data sets taken at different trap frequencies. Dashed vertical lines are drawn at $\tau=2 \pi / \delta$.
Here $\gamma$ is mainly a decay caused by decoherence effects [5], but also includes a contribution due to squeezing. The reduction in fringe visibility due to squeezing ranges from less than $1 \%$ for data set 1 to approximately $9 \%$ for data set 3 . The return time $t_{r}$ constrains the global trajectory, and allows our most direct observations of non-LambDicke behavior, in that we find in general $t_{r}$ is significantly less than $2 \pi / \delta$. The parameter $D$ is related to $\alpha_{0}$, the maximum $\alpha$ which would occur for the same force if the motion were entirely within the LDR. We have

$$
D=R \alpha_{0} \sqrt{2 \bar{n}_{0}+1} \sin \left(\Phi_{w} / 2\right)
$$

where the two trajectories are separated by the angle $\Phi_{w}=$ $\left|\phi_{\uparrow}-\phi_{\downarrow}\right|$ and $R=t_{r} \delta / 2 \pi$ is the fractional reduction in return time.

The phase is fitted by a similarly modified LDR expression, with $B^{2}=R^{2} \alpha_{0}^{2} \sin \left(\Phi_{w}\right)$ :

$$
\phi_{0}(\tau)=(\text { const })+\Delta_{\pi} \tau+B^{2} \sin ^{2}\left(\pi \tau / t_{r}\right) .
$$

With the polarization angles in the experiments, $\Phi_{w}=$ 1.41(5) rad. The amplitude and phase analyses thus each give values of $R \alpha_{0}$ and $t_{r}$. We are then able, using the

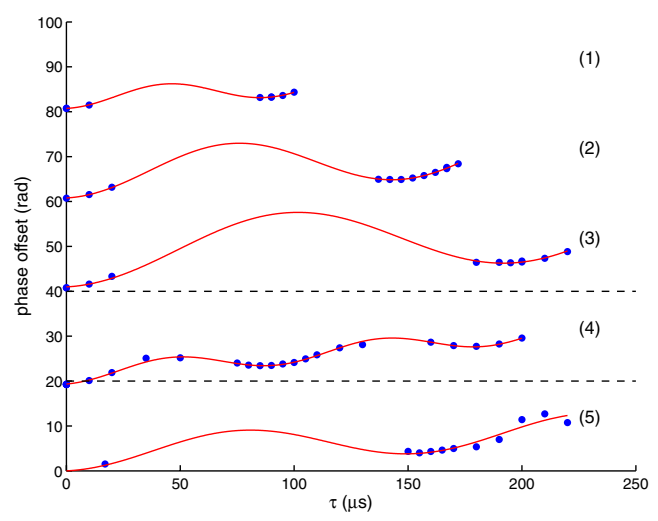

FIG. 3 (color online). Observed phase offset of the fringes as a function of $\tau$, successive data sets are offset by $20 \mathrm{rad}$. 
results of our simulations, to determine $R$ (and hence values for the detuning and $\left.\alpha_{0}\right), \alpha_{\max }$ and $\Delta \alpha_{\max }$. As a check on the validity of our interpretation we can compare the results obtained for $\alpha_{0}$ and $\delta / 2 \pi$ with those expected on the basis of our knowledge of the laser field. The detuning $\delta / 2 \pi$ is known to $\pm 0.5 \mathrm{kHz}$. Two pieces of information quantify the light intensity: the Rabi flopping rate $\Omega_{c}$ when spin-flip ("carrier") transitions are resonantly driven, and the light shift $\Delta_{\pi}$ [deduced from (6)] which comes from off-resonant excitation of these same transitions during the $\mathcal{W}$ pulse.

For all the data there is reasonable agreement between observations and predictions. Sets 1-3 were taken on the same day under particularly stable conditions, and have very good overall consistency. The largest $\alpha_{\max }$ and $\Delta \alpha$ (4.0 and 5.1) were obtained with set 3 . In particular, we note the large reduction in return time $(R=0.67)$. The results of the numerical solution of the TDSE for this case are shown in Fig. 1.

The observed coherence is not perfect, owing mainly to photon scattering and motional effects (dephasing and heating). Let $a \leq 1$ be the predicted overlap of the squeezed states at the return time in a perfect experiment, then $\gamma=\gamma_{s}+\gamma_{m}-\ln (a) / t_{r}$, where $\gamma_{s}$ is caused by the laser pulse $\mathcal{W}$, chiefly by photon scattering, and $\gamma_{m}$ quantifies the decoherence of the mesoscopic superposition itself, chiefly due to motional effects (electric field noise). We see in sets 1-3 behavior consistent with the expected $\Delta \alpha^{2}$ scaling of $\gamma_{m}$ [4,5,9]. We extracted $\gamma_{s}$ from control experiments at large $(200 \mathrm{kHz})$ detuning, where $\gamma_{m}$ is small. After adjusting for the laser beam power used in sets $1-3$, the observed value $\gamma_{s}=1.7(2) \mathrm{ms}^{-1}$ agreed with the photon scattering rate measured in a separate test by detecting spin flips. For data set (3) the TDSE gives $a=$ 0.85 so we obtain $\gamma_{m}=3.0(2) \mathrm{ms}^{-1}$. This is an average over a changing $\Delta \alpha$ : the coherence time $T_{2}=1 / 2 \gamma_{m}=$ 170(10) $\mu$ s for this MSS at its maximum excursion.

Numerical simulations showed that the maximum excursion $\alpha_{\max }$ is a function of only $\eta$ and $\alpha_{0}$. For $\alpha_{0}>1$ a third order polynomial fit of the numerical solutions gave $\alpha_{\max } \simeq\left(0.07683 x^{3}-0.4554 x^{2}+1.135 x-0.01127\right) / \eta$, where $x=\eta \alpha_{0}$. Also, the return time is found to be related to $\alpha_{\max }$ through $1-R \approx 0.82\left(1-\alpha_{\max } / \alpha_{0}\right)$.

We have studied theoretically the forced motion of a quantum oscillator subject to a moving periodic potential, and experimentally demonstrated large MSS's of spin and motion of a trapped ion. The return time reveals the nonuniform nature of the force, and the inferred motion is such that squeezing is confidently expected to be present, though not directly detected. The coherence time is more than an order of magnitude longer than previously reported for this size of mesoscopic superposition, due to the low heating rate in our comparatively large trap. The coherence time is limited by photon scattering and motional heating.
These could be reduced by increasing the detuning of the Raman laser, and increasing the trap size or cooling the electrodes. The issues studied here are also relevant to quantum information experiments where forced motion is used to implement 2-qubit quantum logic gates, and high precision is essential $[12,16,17]$.

This work was supported by EPSRC (QIP IRC), the Royal Society, the European Union, the National Security Agency (NSA), and Disruptive Technology Office (DTO) (No. W911NF-05-1-0297).

[1] M. D. LaHaye et al., Science 304, 74 (2004).

[2] S. Braunstein and P. van Loock, Rev. Mod. Phys. 77, 513 (2005).

[3] C. Monroe, D. M. Meekhof, B.E. King, and D. J. Wineland, Science 272, 1131 (1996).

[4] M. Brune, E. Hagley, J. Dreyer, X. Maître, A. Maali, C. Wunderlich, J.M. Raimond, and S. Haroche, Phys. Rev. Lett. 77, 4887 (1996).

[5] Q. A. Turchette, C. J. Myatt, B.E. King, C. A. Sackett, D. Kielpinski, W. M. Itano, C. Monroe, and D. J. Wineland, Phys. Rev. A 62, 053807 (2000).

[6] Larger MSS's were also created in Refs. [3,7,8], with coherence not observed but likely to be good.

[7] P. C. Haljan, K.-A. Brickman, L. Deslauriers, P. J. Lee, and C. Monroe, Phys. Rev. Lett. 94, 153602 (2005).

[8] A. Auffeves, P. Maioli, T. Meunier, S. Gleyzes, G. Nogues, M. Brune, J. M. Raimond, and S. Haroche, Phys. Rev. Lett. 91, 230405 (2003).

[9] C. J. Myatt, B. E. King, Q. A. Turchette, C. A. Sackett, Kielpinski, W. M. Itano, C. Monroe, and D. J. Wineland, Nature (London) 403, 269 (2000).

[10] S. Wallentowitz and W. Vogel, Phys. Rev. A 55, 4438 (1997).

[11] P. Carruthers and M. M. Nieto, Am. J. Phys. 33, 537 (1965).

[12] D. Leibfried, B. DeMarco, V. Meyer, D. Lucas, M. Barrett, J. Britton, W. M. Itano, B. Jelenkovic, C. Langer, and T. Rosenband et al., Nature (London) 422, 412 (2003).

[13] D. M. Lucas, A. Ramos, J. P. Home, M. J. McDonnell, S. Nakayama, J.-P. Stacey, S.C. Webster, D. N. Stacey, and A. M. Steane, Phys. Rev. A 69, 012711 (2004).

[14] The cooling is by Doppler then Raman sideband cooling; spin state preparation is by optical pumping. $\bar{n}_{0}$ is measured by comparing sideband strengths, cf. F. Diedrich et al., Phys. Rev. Lett. 62, 403 (1989).

[15] M. J. McDonnell, J.-P. Stacey, S. C. Webster, J. P. Home, A. Ramos, D. M. Lucas, D. N. Stacey, and A. M. Steane, Phys. Rev. Lett. 93, 153601 (2004).

[16] D. Leibfried, E. Knill, S. Seidelin, J. Britton, R. B. Blakestad, J. Chiaverini, D. B. Hume, W. M. Itano, J. D. Jost, and C. Langer et al., Nature (London) 438, 639 (2005).

[17] H. Häffner, W. Hänsel, C. F. Roos, J. Benhelm, D. Chekal-kar, M. Chwalla, T. Körber, U. D. Rapol, M. Riebe, and P. O. Schmidt et al., Nature (London) 438, 643 (2005). 